\title{
Designing of $\mathrm{WO}_{3} @ \mathrm{Co}_{3} \mathrm{O}_{4}$ Heterostructure to Enhance Photoelectrochemical Performances
}

Aiymkul A. Markhabayeva ${ }^{t, \neq}$, Md Moniruddint, Robin Dupret, Khabibulla A. Abdullin ${ }^{\ddagger^{*}}$, and Nurxat Nuraje $\mathcal{S}^{*}$

${ }^{\dagger}$ Department of Chemical Engineering, Texas Tech University, Lubbock, TX, USA

‡ National Nanotechnology Laboratory of Open Type (NNLOT), Kazakh National

University, Almaty, 050012 Kazakhstan

§Department of Chemical \& Materials Engineering, Nazarbayev University, Nursultan,

Kazakhstan

*Corresponding authors: Nurxat.nuraje@nu.edu.kz; kh.a.abdullin@mail.ru

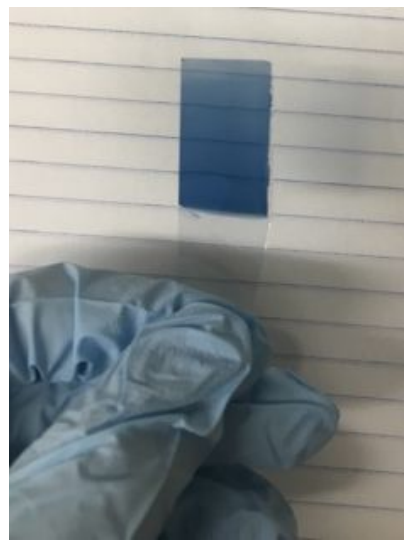

a

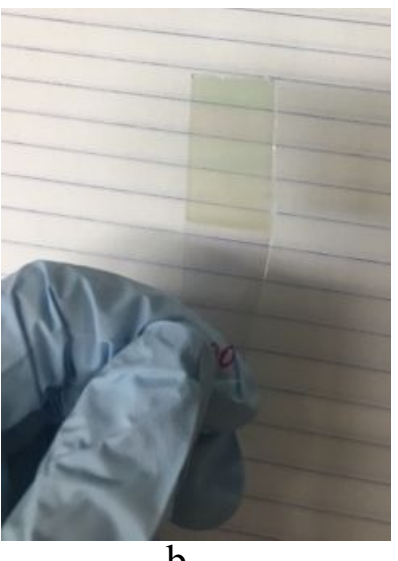

b

Figure S1. Tungsten oxide film a) after deposition and b) after calcination at $500{ }^{\circ} \mathrm{C}$ 


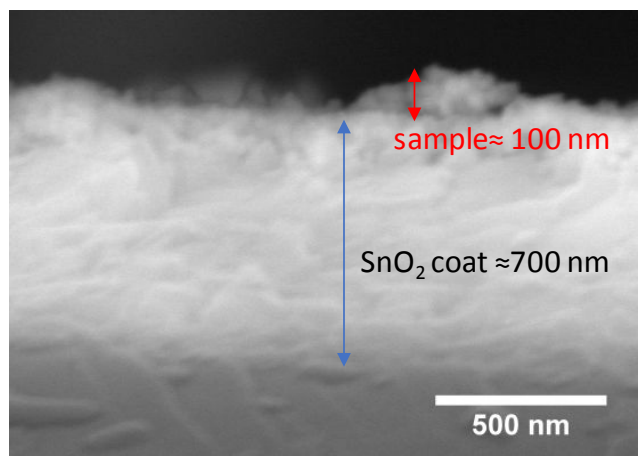

a

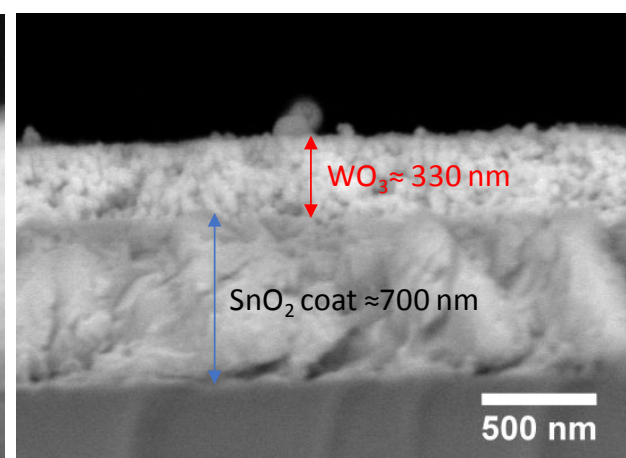

b

Figure S2. Cross section image of $\mathrm{WO}_{3}$ films synthesized during 400 (a) and $600 \mathrm{sec}$ (b) deposition times

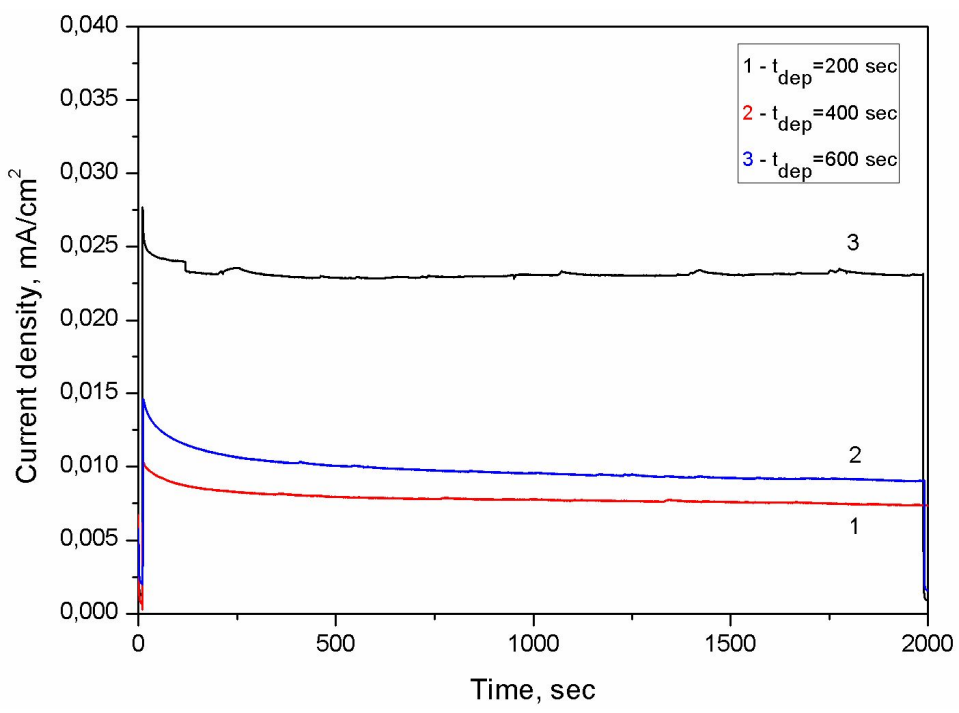

Figure S3. Chronoamperometry at $0.7 \mathrm{~V}$ versus $\mathrm{Ag} / \mathrm{AgCl}$ : $\mathrm{WO}_{3}$ electrodes with different deposition times 


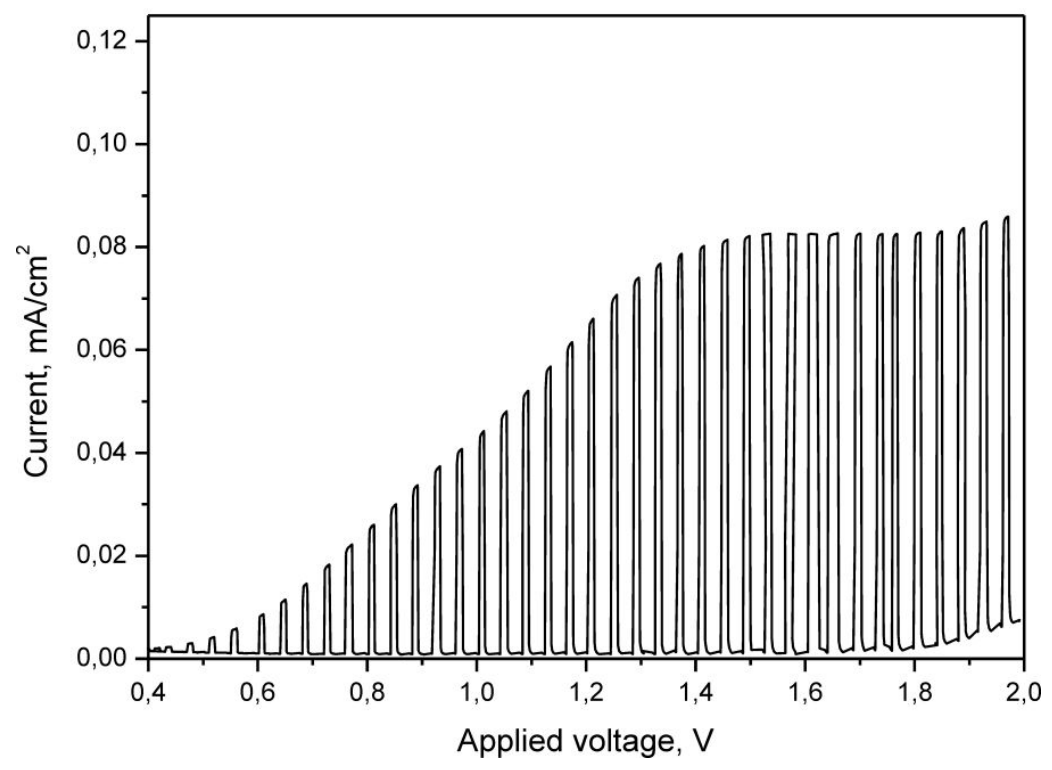

Figure S4. J-V curves with chopped illumination for $\mathrm{WO}_{3}$ films

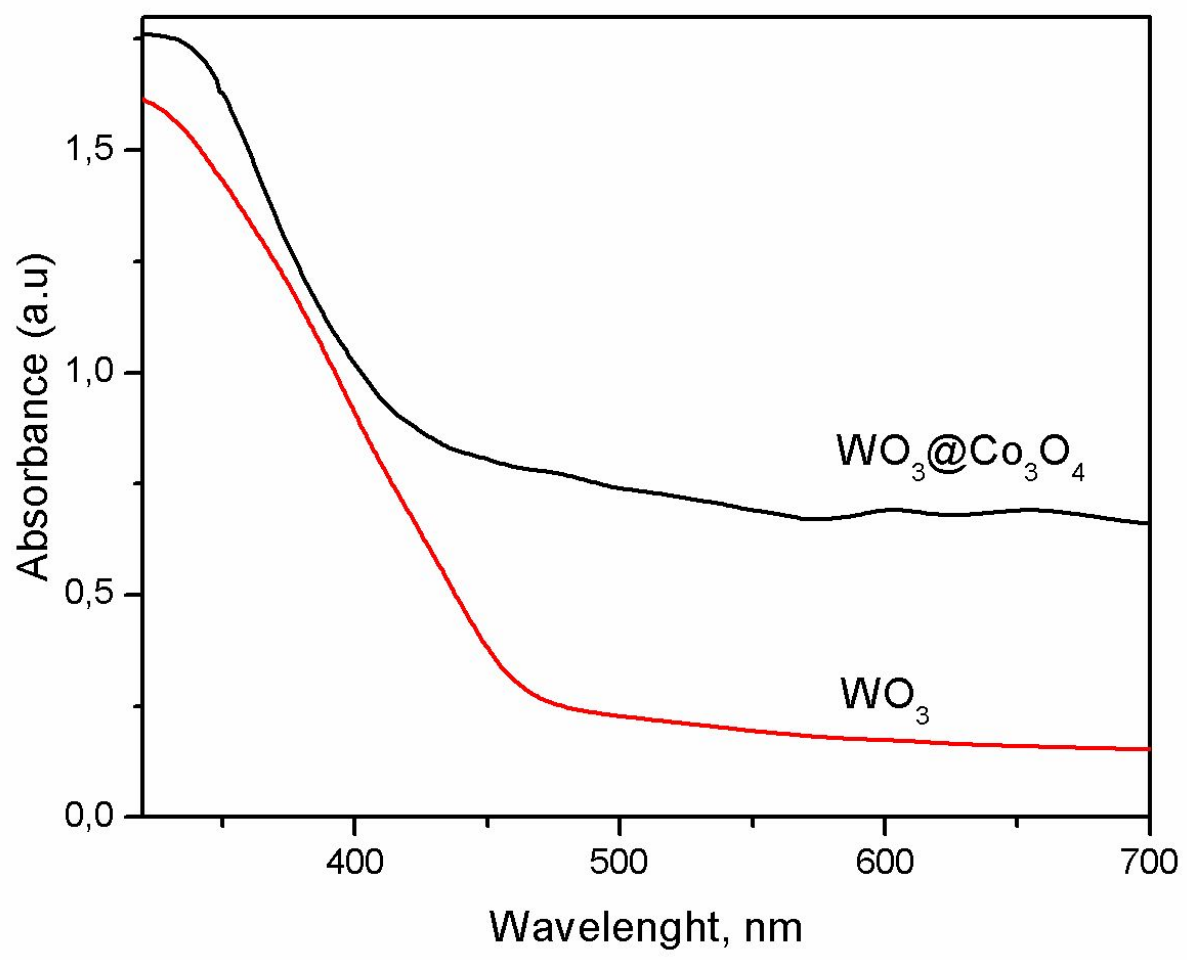

Figure S5- UV-Vis diffuse reflectance spectra (DRS) of WO3 and WO3@Co3O4 